\title{
Regulation of Distinct Attractive and Aversive Mechanisms Mediating Benzaldehyde Chemotaxis in Caenorhabditis elegans
}

\author{
William M. Nuttley, ${ }^{1}$ Singh Harbinder, and Derek van der Kooy \\ Department of Anatomy and Cell Biology, University of Toronto, Toronto, Ontario M5S 1A8, Canada
}

\begin{abstract}
Olfactory-mediated chemotaxis in nematodes provides a relatively simple system to study biological mechanisms of information processing. Analysis of the kinetics of chemotaxis in response to $100 \%$ benzaldehyde revealed an initial attractive response that is followed by a strong aversion to the odorant. We show that this behavior is mediated by two genetically separable attraction- and aversion-mediating response pathways. The attraction initially dominates behavior but with prolonged exposure habituation leads to a behavioral change, such that the odorant becomes repulsive. This olfactory habituation is susceptible to dishabituation, thereby re-establishing the attractive response to the odorant. Re-examination of the putative olfactory adaptation mutant adp-1(ky20) revealed that the phenotype observed in this line is due to a supersensitivity to a dishabituating stimulus, rather than a defect in the adaptation to odorants per se. A modified benzaldehyde chemotaxis assay was developed and used for the isolation of a mutant with a specific defect in habituation kinetics, expressed as a persistence of the attractive response.
\end{abstract}

The ability to sense and respond to environmental stimuli is essential for the survival of animals. Responses to chemical cues have been observed in systems ranging from bacteria to mammalian cells, with olfaction being widely employed. Olfactory responses in a number of organisms exhibit plasticity, such that an initially robust response is diminished after prolonged exposure. Two general processes have been described to account for this behavior. Olfactory adaptation is the attenuation of a specific odorant response pathway as a result of prolonged pre-exposure and requires a rest period for the naive response to recover. Habituation also results in a reduced response to a stimulus but differs from adaptation in that dishabituating stimuli can result in a rapid recovery to naive response levels. Dishabituation is a hallmark, but not the only characteristic of the habituation process, and implies a potentially more complex response in which environmental events cause the rapid reinstatement of the previous, naive response levels (Groves and Thompson 1970; Jorgensen and Rankin 1997). The olfactory behavior displayed by the nematode Caenorhabditis elegans presents a relatively simple and well-characterized system with which to investigate the mechanisms underlying behavioral plasticity.

The canonical odorant receptor in nematodes, the diacetyl receptor, is the product of the odr-10 gene and a member of a large family of similar heterotrimeric G-protein

${ }^{1}$ Corresponding author.

E-MAIL bill.nuttley@utoronto.ca; FAX (416) 978-3844.

Article and publication are at www.learnmem.org/cgi/doi/ $10.1101 / \mathrm{lm} .36501$. coupled receptors (GPCRs) found in C. elegans (Sengupta et al. 1996; Bargmann 1998; Robertson 1998). Twenty-four genes encoding heterotrimeric G-protein subunits, effectors of GPCR signaling, have also been identified, and their products are involved in a number of processes including chemotaxis, thermotaxis, egg-laying, and entry and exit from the dauer larval stage (Bargmann 1998; Bargmann and Kaplan 1998; Jansen et al. 1999). At the neuroanatomical level, the six primary olfactory neurons (the AWA, AWB, and AWC neuron pairs) mediate attractive and repulsive responses to $>40$ different volatile chemicals, with most odorants being detected by a single neuron pair (Bargmann et al. 1993). Additionally, the ASH and ADL neurons mediate the aversive responses to a number of stimuli, including some volatile odorants (Troemel et al. 1995; Bargmann and Mori 1997). Not only can a single neuron distinguish many different chemicals, but the animals are capable of adapting to a single chemical while maintaining their sensitivity to others that are detected by the same primary sensory neuron (Colbert and Bargmann 1995). This complexity makes C. elegans an attractive model with which to study the molecular basis of neural information processing and plasticity (Jorgensen and Rankin 1997; Bargmann and Kaplan 1998).

The idea that individual neurons mediate either attractive or aversive responses is supported by heterologous expression studies with the diacetyl receptor odr-10. Troemel et al. (1997) demonstrated that expression of the odr-10 gene in the aversion-mediating, AWB, amphid neurons (odr10 is expressed in the AWA neurons in wild-type animals) caused the animals to avoid, rather than approach, the odor-

LEARNING \& MEMORY 8:170-181 @ 2001 by Cold Spring Harbor Laboratory Press ISSN1072-0502/01 \$5.00

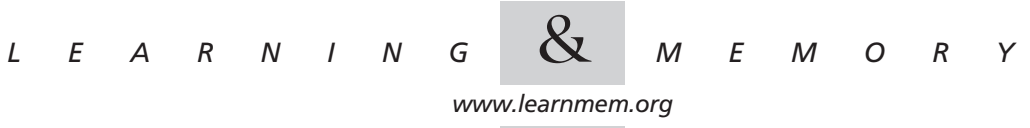


ant. This result suggested that the response to an odorant depends primarily on whether its receptor is expressed on an attraction-mediating or an aversion-mediating sensory neuron. However, some odorants, such as benzaldehyde and 2,4,5-trimethylthiazole, have been reported to act as attractants at low concentrations but repellents at high concentrations (Bargmann et al. 1993). We have noticed that naive nematodes are attracted to high concentrations of benzaldehyde and that the aversion to $100 \%$ benzaldehyde seen in standard chemotactic assays develops as a result of the odorant exposure during the 1-h test. Furthermore, animals that have developed an aversion to a high concentration of benzaldehyde will express this aversion when tested with lower concentrations (1\%) of the odorant, which are attractive for naive animals. In this report, we present a detailed analysis of benzaldehyde chemotaxis in C. elegans, which is shown to involve separate attractive and repulsive response mechanisms. We introduce a simple model of the observed behavioral plasticity based on the dynamic interaction of these two opposing, benzaldehyde-sensitive processes and investigate a number of predictions of this model. We also present evidence that the previously described olfactory adaptation is susceptible to dishabituation and show that the $a d p-1(k y 20)$ mutation acts by increasing the animals' sensitivity to dishabituating stimuli. This observation led to the development of a modified behavioral assay for identifying novel adaptation mutants.

\section{RESULTS}

\section{Chemotactic Response to Benzaldehyde}

The volatile chemical benzaldehyde elicits a robust chemotactic response from $C$. elegans. When tested in standard chemotactic assays, benzaldehyde was an attractant over a wide concentration range as are a number of odorants (data not shown; Bargmann et al. 1993). When presented with a $100 \%$ point source, however, the animals showed an aversion after a 1-h test. This biphasic type of dose-response relationship is not observed with most other odorants, such as diacetyl, which acts as an attractant at all concentrations (data not shown; Bargmann et al. 1993). We have investigated the kinetics of benzaldehyde chemotaxis and found that aversion is preceded by a strong, transient approach response to $100 \%$ benzaldehyde. When 100-200 animals were tested on a single plate, the population chemotactic index (C.I.) peaked within $20 \mathrm{~min}$, then slowly decreased (Fig. 1A; 100\%). It is notable that the animals did not swim right up to the point source, but instead they maintained a minimum separation of about $1 \mathrm{~cm}$ from the drop of benzaldehyde. After $90 \mathrm{~min}$ of exposure, the C.I. was negative, indicating that the animals were avoiding the benzaldehyde. This was not due simply to randomization on the plate after adaptation of the benzaldehyde attraction, as $>90 \%$ of the
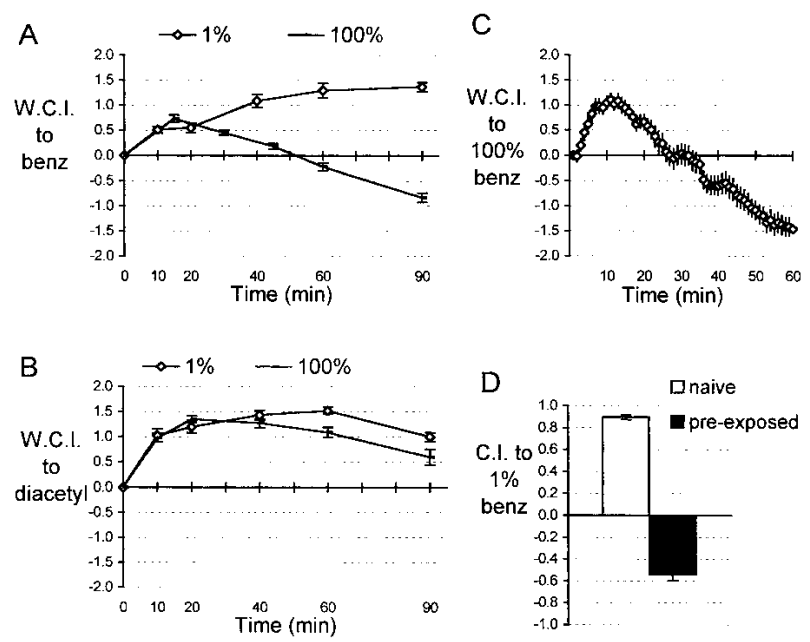

Figure 1 Chemotaxis by Caenorhabditis elegans. (A) Time course for approach to $100 \%$ ( $n=8$ plates per point) and $1 \%(n=14$ plates per point) benzaldehyde. (B) Time course for approach to $100 \%$ and $1 \%$ diacetyl ( $n=12-14$ plates for all points). (C) Time course for approach to $100 \%$ benzaldehyde by animals tested individually ( $n=40$ animals). ( $D$ ) One-hour approach to $1 \%$ benzaldehyde by naive animals and those with a 90-min pre-exposure to $100 \%$ benzaldehyde ( $n=10$ plates per point). A positive W.C.I. or C.I. indicates an attraction to the odor; negative score indicates an aversion.

animals on the plate after $90 \mathrm{~min}$ were on the half of the plate furthest from the benzaldehyde spot.

When animals were presented with a spot of $100 \%$ diacetyl rather than benzaldehyde, they showed a similar initial attraction but not a subsequent aversion (Fig. 1B). Instead, after prolonged exposure to diacetyl, the nematodes began to randomize on the test plate, presumably due to adaptation of the attractive response to diacetyl (Colbert and Bargmann 1995). When tested with $1 \%$ diacetyl, the behavior was qualitatively similar but the switch from a positive slope to a negative slope (at the C.I. maximum) was delayed, indicating that adaptation (expressed as a decreasing C.I.) also occurs in the presence of $1 \%$ diacetyl but at a slower rate. Interestingly, there was no evidence of adaptation (decreasing C.I.) after prolonged exposure to $1 \%$ benzaldehyde (Fig. 1A), but rather, the animals remained at the odorant spot once they located it. This result also indicates that this dose of the odorant is insufficient to induce the behavioral response seen in the presence of $100 \%$ benzaldehyde.

A qualitatively similar response to $100 \%$ benzaldehyde was seen whether animals were tested in groups or individually (Fig. 1C). A quantitative difference was observed, however, in that the time required for the mean C.I. to cross into negative values was about $30 \mathrm{~min}$ for individually tested animals rather than the 45-60 min typically seen in population assays. This result may be due to the interactions between individuals during population assays. Time-lapse video photography has revealed that aggregates of 5-30

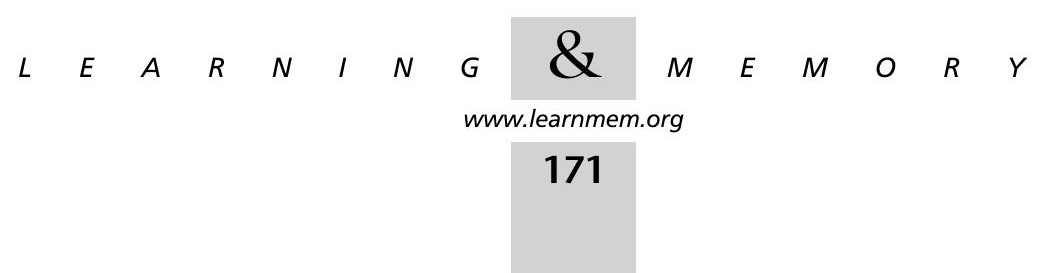


animals formed and dispersed throughout the course of the assay (not shown). These interactions appeared to delay the response kinetics while not interfering with the overall pattern of an attractive phase followed by an aversion.

Previous studies of olfactory adaptation in C. elegans have shown that after a prolonged pre-exposure to high odorant concentrations, nematodes show a reduced attraction to a lower concentration of the same odorant, including benzaldehyde (Colbert and Bargmann 1995; and see below). Because the animals actually developed a strong aversion to $100 \%$ benzaldehyde after $90 \mathrm{~min}$ of exposure, we wondered whether this aversion would generalize to a lower concentration of the odorant. Therefore, we tested whether the aversive response, induced by a 90-min preexposure to $100 \%$ benzaldehyde, could be detected in the presence of $1 \%$ benzaldehyde. Animals in groups of $600-$ 800 were pre-exposed to $2 \mu \mathrm{L}$ of $100 \%$ benzaldehyde for 90 min then collected in $2 \mathrm{~mL}$ of water and transferred to new chemotaxis plates and tested for their response to a $1 \mu \mathrm{L}$ spot of $1 \%$ benzaldehyde. As shown in Figure 1D, after the pre-exposure, the animals displayed a strong aversive response (pre-exposed, C.I. $=-0.55 \pm 0.05$ ) to the test odorant, in contrast to the attraction displayed by the naive animals (C.I. $=0.89 \pm 0.02$ ) in these experiments. This difference demonstrates that a low concentration of benzaldehyde can be aversive under some conditions, although prolonged exposure to $1 \%$ benzaldehyde cannot in itself induce the aversion. This result indicates that different minimum stimulus levels are required for the induction of, versus the expression of, the aversive chemotactic response.

The complex response to $100 \%$ benzaldehyde could result from the interplay of two opposing response pathways. We suggest a working model (Fig. 2) in which the response of naive animals is dominated by an attractionmediating pathway. With prolonged exposure, either the adaptation of this response, and/or the strengthening of an aversion-mediating pathway, shifts the balance such that the aversive response predominates. A number of mutant alleles have been described that allowed us to investigate various aspects of this model.

\section{Genetic Dissection of the Attractive and Aversive Components of Benzaldehyde Chemotaxis}

Chemotaxis to volatile odorants, including benzaldehyde, has been studied in great detail. The amphid wing neuron pairs AWA and AWC mediate the attractive response to a number of volatile odorants. Laser ablation studies have indicated that the AWC neurons are required for chemotaxis to low concentrations of benzaldehyde and a number of chemotaxis-deficient mutant lines have been identified that have deficits in their approach to a dilute benzaldehyde source (Bargmann et al. 1993). These include chemotaxis (che and $\operatorname{tax}$ ) and odorant-response (odr) mutants, all of

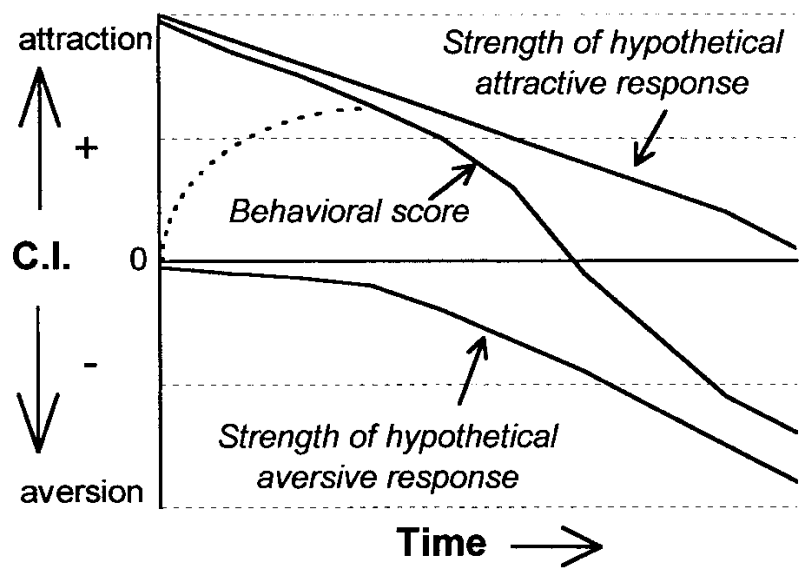

Figure 2 Working model showing how the interaction of opposing attractive and aversive processes could sum to yield the chemotactic response that is observed. The dotted line connecting the origin to the hypothetical response line depicts the observed response scores, this deviation from the hypothetical sum reflects the placement of the animals in the center of the plate at $t=0$ and the time taken for them to initially move toward the benzaldehyde.

which have defects in additional chemotactic responses. Three neuron pairs that are known to mediate aversive responses to volatile chemicals are AWB, ADL, and ASH. The ASH neurons are polymodal sensory neurons involved in a number of aversive responses, including nose touch avoidance (Kaplan and Horvitz 1993), osmotic avoidance (Bargmann et al. 1990), and avoidance of some volatile odorants, including benzaldehyde (Troemel et al. 1995). A number of mutant lines with defective responses to benzaldehyde were tested for their performance in our kinetic assay, and the results for two particularly interesting lines are shown in Figure 3.

The odr-3(n2150) mutant, which was isolated initially because of a low approach to $1 \%$ benzaldehyde, identified a gene that encodes a G-protein $\alpha$ subunit that is required for behavioral responses known to be mediated by the AWA, AWB, AWC, and ASH sensory neurons (Troemel et al. 1997; Roayaie et al. 1998) and is also expressed in the ADF neurons. There was a large reduction in the approach scores when these animals were tested with 1\% (data not shown; Roayaie et al. 1998) or 100\% benzaldehyde (Fig. 3A, 15- and 30 -min points). However, the scores at 60 and 90 min show that this mutant strain retains the strong wild-type aversion to $100 \%$ benzaldehyde. Single-animal analysis (Fig. 3B) also revealed the absence of a robust attractive response compared with wild-type nematodes (Fig. 1C). A two-factor analysis of variance (ANOVA) with the single-animal data revealed a significant time $\mathrm{X}$ strain interaction $\left(F_{1,59}=10.3\right.$, $P<0.05)$. Separate analyses for the two halves of the assay showed a significant interaction over the first $30 \mathrm{~min}$ $\left(F_{1,29}=5.5, P<0.05\right)$, but not over the second $30 \mathrm{~min}$ $\left(F_{1,29}=0.7, P>0.05\right)$, confirming that the difference was expressed during the initial, attractive phase of the re-

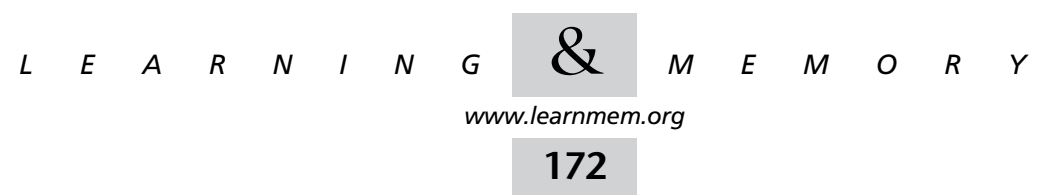



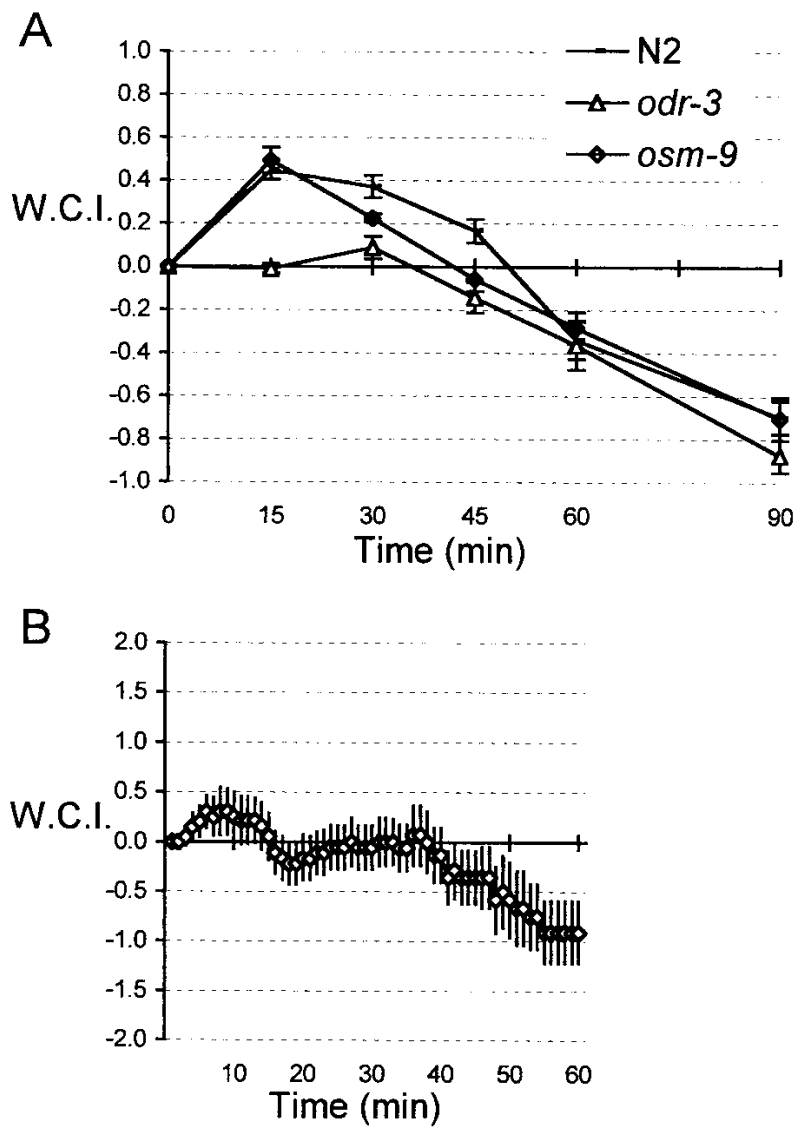

Figure 3 Dissociation of the attractive and aversive responses. (A) Chemotactic behavior of N2, odr-3(n2150), and osm-9(ky10) animals in the presence of $100 \%$ benzaldehyde (from left: for N2, $n=12,11,14,12$, and 16 plates; for odr-3(n2150), $n=15,6,11$, 7, and 9 plates; for osm-9(ky10), $n=4,6,10,13$, and 6 plates). (B) Chemotaxis by individual odr-3(n2150) animals in response to $100 \%$ benzaldehyde ( $n=29$ animals).

sponse. This genetic dissection of the attractive and aversive responses to benzaldehyde seen in odr-3(n2150) mutants indicates that these behaviors may be mediated by distinct neuronal systems.

The data from odr-3(n2150) mutants also reveal some interesting characteristics of the pathway mediating the aversion. As shown in Figure 3A, there is no indication of an aversion to $100 \%$ benzaldehyde at the 15 - and 30-min time points in the population assay. This observation indicates that the aversive response measured in this assay is not immediately expressed in naive animals even in the absence of an attractive response, but rather its expression requires some pre-exposure to benzaldehyde. Additionally, no aversion was seen during the first $30 \mathrm{~min}$ of the single animal assay with $o d r-3(n 2150$ ) (Fig. 3B), supporting the notion that the chemotactic aversion to benzaldehyde requires a period of pre-exposure to the odorant to be expressed behaviorally. A delay in the onset of the aversion relative to $\mathrm{N} 2$ was also seen in the population assay (Fig 3A). The trace for the N2 and osm-9(ky10) animals has a negative slope between 15 and $30 \mathrm{~min}$, indicating movement away from the source, whereas the $o d r-3(n 2150)$ animals do not display a negative slope until the $30-$ to 45 -min time window. The same trend was observed in the single animal assay (cf. Figs. $3 \mathrm{~B}$ and $1 \mathrm{C}$ ) with the wild-type, N2, animals developing a negative slope earlier than the odr-3(n2150) animals, which also showed a delay of about $10 \mathrm{~min}$, from 30 to 40 min, for crossing into a negative C.I. value. Presumably, the lack of an initial attraction in the odr-3(n2150) animals ensures that they do not get as close to the benzaldehyde as the wild-type animals do at earlier time points in the assay. This situation would cause the mutants to experience a lower dose of benzaldehyde at these early time points, resulting in a slightly slower induction of the aversion mediating pathway. Indeed, in population tests when the odr$3(n 2150)$ animals were placed closer to the spot of $100 \%$ benzaldehyde, an aversive response was detected within 20 min of exposure onset (not shown).

The osm-9(ky10) allele disrupts a number of nematode behaviors, including all responses known to be mediated by the ASH neurons, presumably due to the proposed role of the OSM-9 protein in modulating membrane permeability. Both genetic and laser ablation experiments have indicated that the ASH neurons are involved in short-range benzaldehyde-mediated avoidance responses (Troemel et al. 1995; Colbert et al. 1997). The similarity between the predicted primary structure of the putative OSM9 protein and the mammalian capsaicin receptor (Tominaga et al. 1998) has strengthened the case for the designation of ASH as a nociceptive neuron in C. elegans (Driscoll and Kaplan 1998). Additionally, the osm-9 gene is expressed in ADL, which also mediates aversive responses to volatile odorants (Troemel et al. 1995), as well as in additional sensory cells and some non-neuronal cells (Colbert et al. 1997). The osm9(ky10) mutant strain showed wild-type chemotactic behavior in our chemotaxis assay (Fig. 3A), demonstrating that neither osm-9 nor odr-3 is required for the benzaldehydeinduced aversive response measured here.

\section{The adp-1(ky20) Mutation \\ Facilitates Dishabituation}

The attractive response to a large number of odorants adapts over time such that pre-exposure to an odorant reduces the specific approach response to that odorant on a subsequent test (Colbert and Bargmann 1995). The CX20 adp-1(ky20) strain is deficient in its adaptation to benzaldehyde, although naive approach behavior is unaffected (Fig. 4A; Colbert and Bargmann 1995). Our model (Fig. 2) predicts that this type of mutation would interfere with the kinetics of benzaldehyde chemotaxis. However, the adp1(ky20) mutation was found not to impair the behavior of the animals in the kinetic analysis, as they acted similar to the wild-type N2 strain throughout the assay (Fig. 4B).

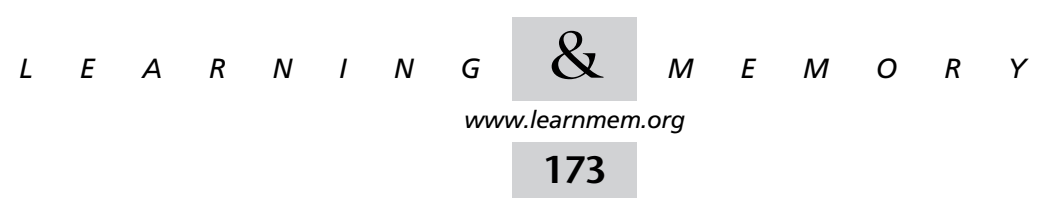


A

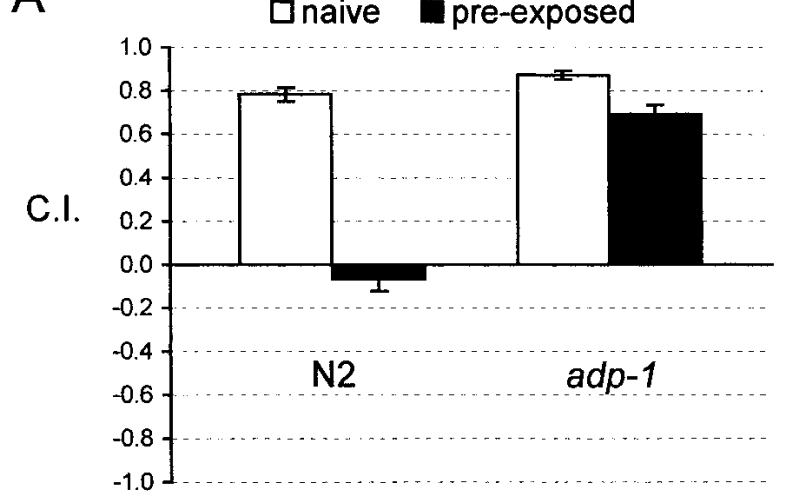

B

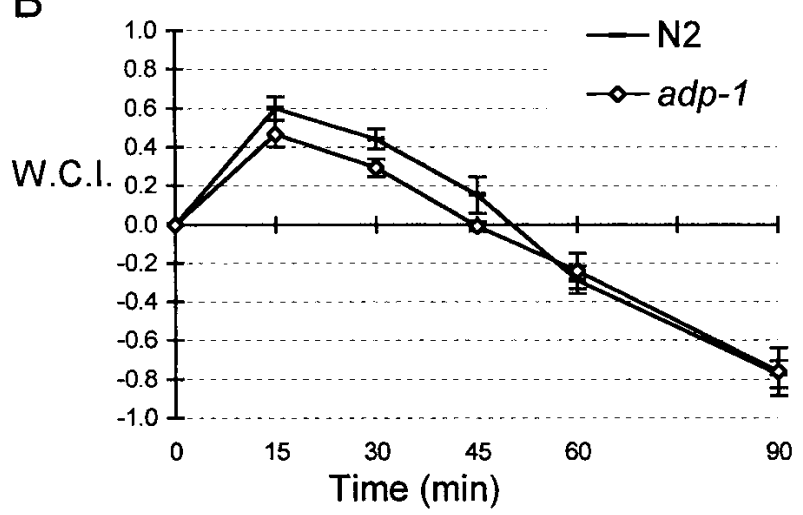

Figure 4 Adaptation to benzaldehyde. (A) Approach to 1\% benzaldehyde by naive animals and those that had been pre-exposed to $100 \%$ benzaldehyde for $1 \mathrm{~h}$ immediately before testing. $(n=10$ plates for naives and 13 plates for pre-exposed). (B) Chemotaxis to $100 \%$ benzaldehyde by N2 and adp-1 (ky20) mutant animals (From left: for N2, $n=12,4,4,7$, and 7 plates; for adp-1(ky20), $n=4,3$, 2,7 , and 7 plates).

Therefore, although adp-1(ky20) animals do not adapt to benzaldehyde in the traditional assay, this strain retains the behavioral plasticity required to produce a wild-type response in the kinetic assay. One possible explanation for this apparent inconsistency is that, during tests for adaptation, the transfer of the animals from the adaptation plate to the test plate may act as a dishabituating stimulus. In contrast, in the kinetic assay, the animals stay on the same plate throughout the test session.

To investigate whether the handling of the animals had an effect on subsequent chemotaxis scores, we performed a modified adaptation assay in which the animals were adapted and tested on the same plates, thereby eliminating the transfer to a different plate for testing (see Materials and Methods). Naive animals from both wild-type and adp1(ky20) strains showed equivalent responses to both $1 \%$ and $100 \%$ benzaldehyde (Fig. 5A). After a 1-h pre-exposure to $100 \%$ benzaldehyde, the responses of adp-1(ky20) animals were indistinguishable from the wild-type N2 animals on subsequent approach tests to either 1\% or $100 \%$ benzaldehyde, but only when they were tested on the same plate that they received the pre-exposure. After transfer to a fresh plate for testing the $a d p-1(k y 20)$ mutant strain recovered significantly (Fig. 5A; same compared with different plates for test to $1 \%\left[t_{11}=8.4, P<0.05\right]$ or $100 \%$ $\left[t_{12}=7.4, P<0.05\right]$ benzaldehyde). These data confirm that the handling of the adp-1(ky20) mutant animals in standard adaptation assays has a profound effect on subsequent behavioral scores.

\section{Dishabituation of Benzaldehyde Adaptation}

The observation that the adp-1(ky20) mutation does not significantly affect the behavior of the animals in the kinetic assay of benzaldehyde chemotaxis suggests that the deficit caused by this allele does not interfere with the response decrement induced by pre-exposure to benzaldehyde. Rather, the mutation appears to affect the response only after the animals have been handled (Fig. 5A). This observation suggests that the transfer of the adp-1(ky20) animals between plates acted as a dishabituating stimulus for these animals. Interestingly, a slight but significant $\left(t_{11}=3.1\right.$, $P<0.05)$ increase in the approach to $100 \%$ benzaldehyde by wild-type animals was also observed after transfer to fresh (different) plates in comparison with use of the same plates for testing (Fig. 5A, right), although their scores remained well below those of the adp-1(ky20) mutants $\left(t_{5}=7.2, P<0.05\right)$. As dishabituation after benzaldehyde adaptation has not been reported previously in C. elegans, we investigated the effect on wild-type, N2 animals of harsher treatment between the adaptation and test stages of the assay. In the standard assay, we transfer the animals to a test tube in $\sim 2 \mathrm{~mL}$ of water and let them settle without centrifugation. The animals from the bottom of the tube were then removed and applied to a test plate (Fig. 5B, habituated condition). To test for dishabituation, the wildtype animals were instead collected in $10 \mathrm{~mL}$ of water and centrifuged at a relative centrifugal force (RCF) of $65 g$ for 1 min. The resultant pellet was then resuspended in $10 \mathrm{~mL}$ of water and recentrifuged at $65 \mathrm{~g}$ for $2 \mathrm{~min}$. When the animals were then tested for their approach to benzaldehyde, much of the response decrement was abolished (Fig. 5B, dishabituated condition). In control experiments, both the volume of the dishabituation washes $(20 \mathrm{~mL}$ or $2 \mathrm{~mL}$ total) and force applied to sediment the animals $(\mathrm{RCF}=65 \mathrm{~g}, 16 \mathrm{~g}$, or $1 g$ ) were varied. As shown in Figure 5C, reducing either the volume or the RCF resulted in a significant reduction in the test C.I.s compared with the dishabituated animals, suggesting that these factors work synergistically to achieve the level of dishabituation seen with two 10-mL washes and high speed sedimentation at $65 g$ (Fig. 5C).

To investigate further this dishabituation phenomenon, we performed habituation experiments using a range of concentrations from $1 \%$ to $100 \%$ for the habituating stimu-

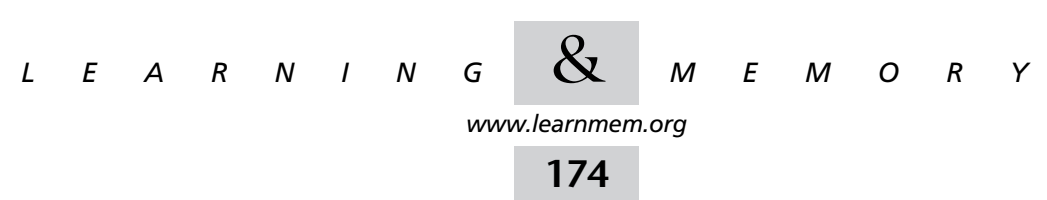



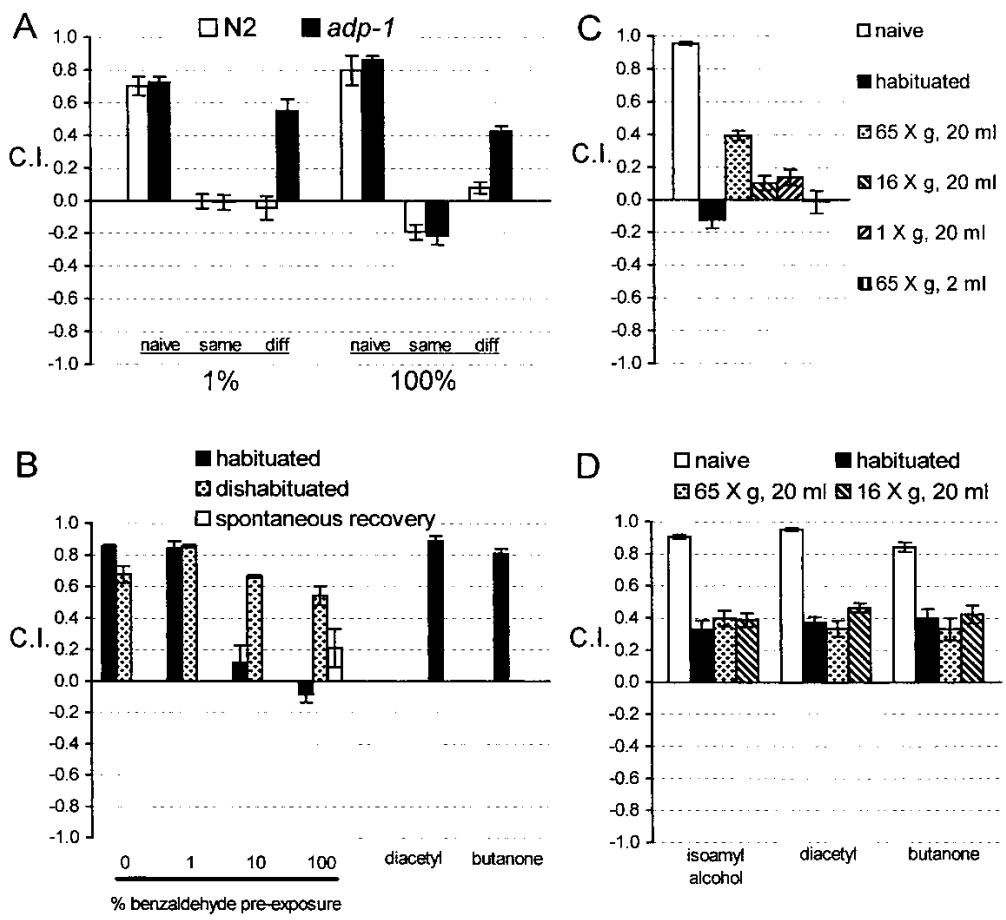

Figure 5 Dishabituation of benzaldehyde adaptation. (A) Single-plate assay for adaptation. Animals pre-exposed to $2 \mu \mathrm{L}$ of $100 \%$ benzaldehyde for $1 \mathrm{~h}$ were assayed for their 30-min (1\%) or 20-min (100\%) approach to benzaldehyde either on the same plate on which they were pre-exposed (same) or after gentle transfer to a fresh plate for testing (different). Scores for naive animals are shown for comparison, (from left: for N2, $n=3,6,6,3,10$, and 3 plates; for adp-1(ky20), $n=4,6,7,4,10$, and 4 plates). (B) $\mathrm{N} 2$ animals were exposed for $1 \mathrm{~h}$ to $2 \mu \mathrm{L}$ of odorant then transferred to fresh plates to test approaches to $1 \%$ benzaldehyde. Habituated, dishabituated, and spontaneous recovery groups were treated as described in the text (from left, $n=14,3,3,3,6,3,17,11,3,3$, and 3 plates). The final two bars show chemotaxis to $1 \%$ benzaldehyde by animals that had been pre-exposed for $1 \mathrm{~h}$ to either diacetyl or butanone. (C) N2 animals were exposed for $1 \mathrm{~h}$ to $2 \mu \mathrm{L}$ of odorant then transferred to fresh plates to test approaches to $1 \%$ benzaldehyde. In addition to the regular transfer conditions (habituated; transferred in $2 \mathrm{~mL}$ of water and settled at $1 \mathrm{~g}$ ), four different transfer protocols were followed, varying the RCF $(1 g$, $16 g$, or $65 g)$ used to sediment the animals and the volume of the wash ( 2 or $20 \mathrm{~mL}$ total; from left, $n=14$, $21,6,15,12$, and 8 plates). (D) Animals were pre-exposed to $2 \mu \mathrm{L}$ of odorant for 1 $\mathrm{h}$ then tested after a gentle transfer in $2 \mathrm{~mL}$ of water or dishabituation by centrifugation twice at either $65 \mathrm{~g}$ or $16 \mathrm{~g}$ in $10 \mathrm{~mL}$ of water. Tests were $1-\mathrm{h}$ approaches to $0.1 \%$ diacetyl and isoamyl alcohol and to $0.01 \%$ butanone (from left, $n=13,18,6$, $12,13,18,6,12,9,9,6$, and 3 plates).

lus. The degree of habituation increased proportionally to the strength of the habituating stimulus (Fig. 5B), as was reported in previous studies on olfactory adaptation in $C$. elegans (Colbert and Bargmann 1995). Significantly higher C.I.s were achieved by dishabituated compared with habituated animals after pre-exposure to either $10 \%\left(t_{7}=3.3\right.$, $P<0.05)$ or $100 \%\left(t_{26}=8.3, P<0.05\right)$ benzaldehyde. Importantly, the scores for these dishabituated animals were similar to those of control animals that received no habituating stimulus but did experience the centrifugation step for dishabituation (Fig. 5B; 0\% benzaldehyde pre-exposure, white bar). When the animals were kept for $90 \mathrm{~min}$ on food between the adaptation and testing phases of the assay, an intermediate degree of attraction was observed (Fig. 5B; spontaneous recovery). This level of spontaneous recovery is still significantly lower $\left(t_{12}=2.9, P<0.05\right)$ than that achieved after the 3-min dishabituation procedure. Indeed, it has been shown that full recovery of benzaldehyde sensitivity takes about $3 \mathrm{~h}$ under these conditions (Colbert and Bargmann 1995). Preexposure to diacetyl or butanone did not affect the approach to benzaldehyde (Fig. 5B), and a similar lack of cross habituation was found when benzaldehyde-habituated animals were tested for their approach to butanone or diacetyl (data not shown; Colbert and Bargmann 1995). Taken together, these results demonstrate that benzaldehyde-specific adaptation in wild-type animals is susceptible to dishabituation and that the adp-1(ky20) mutation renders the animals supersensitive to dishabituating stimuli. This apparent gain of function is consistent with the dominant nature of the adp-1(ky20) allele.

The effect of the dishabituation protocol was tested after pre-exposure to either diacetyl isoamyl alcohol, or butanone (Fig. 5D). Each odorant was tested at the concentration that showed the largest reduction in C.I. after a 1-h pre-exposure by inversion over a $2-\mu \mathrm{L}$ drop of the undiluted chemical. All three chemicals were able to produce a significant suppression of their attractive response. However, this muted response was the same whether the animals were transferred to the test plates by either the gentlest $(1 g, 2 \mathrm{~mL})$ or the harshest $(65 g, 20 \mathrm{~mL})$ procedures used.

The specificity of the dishabituation protocol, affecting benzaldehyde- but not butanone-, isoamyl alcohol-, or diacetyl-treated animals, may explain why previous studies of olfactory adaptation in C. elegans (Colbert and Bargmann 1995, 1997) have shown higher approach scores to benzaldehyde after pre-exposure treatments than reported here. In those studies, the nematodes were washed four times by high-speed centrifugation between the pre-exposure and test phases of the experiments, and this step would have resulted in a significant dishabituation of the benzaldehyde-treated animals. In contrast, the postexposure scores we observed for animals treated with either butanone, isoamyl alcohol, or diacetyl (Fig. 5D) were comparable with those reported in previous studies (C.I. range of 0.2 to 0.5; Colbert and Bargmann 1995, 1997), consistent with the lack of dishabituation observed after pre-exposure to these odorants (Fig. 5D).

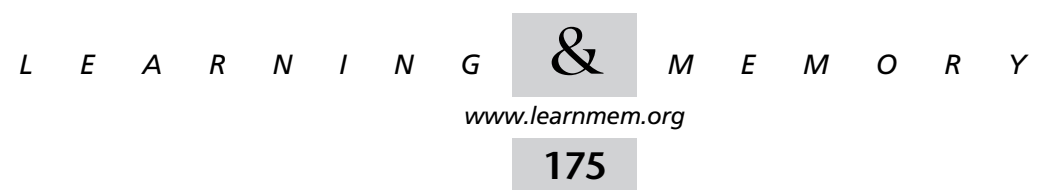




\section{Chemotaxis and Dishabituation Defects in eat-4(ky5) Mutants}

The eat 4 gene encodes a protein homologous to rat brainspecific sodium inorganic phosphate cotransporter I (BNPI; Lee et al. 1999), and eat-4(ky5) mutants are defective in both the habituation of the tap-response and the electric shock-induced dishabituation after tap-response habituation (Rankin and Wicks 2000). Therefore, we tested the performance of eat-4(ky5) animals in benzaldehyde chemotaxis and learning. Although eat-4(ky5) animals showed a significant deficit in their naive approach level to $1 \%$ benzaldehyde (Fig. 6), they still displayed significant habituation of the attraction after a $1-\mathrm{h}$ exposure to $100 \%$ benzaldehyde. This habituated response was, however, refractory to dishabituation, as the habituated eat-4(ky5) animals did not approach benzaldehyde after they were centrifuged twice at $65 \mathrm{~g}$. Therefore, eat-4(ky5) mutants are defective in two quite unrelated dishabituation protocols, electric shock after tap-response habituation and centrifugation after olfactory habituation.

\section{Aversion Onset Kinetics as a Screen for Benzaldehyde Habituation Mutants}

The finding that the phenotype observed with adp-1(ky20) animals is a result of dishabituation suggested that a singleplate assay could provide a useful screen to identify animals with defects in nonassociative learning, in particular in the response decrement phase of the habituation process and the switch to an aversive response. The $\mathrm{F}_{2}$ progeny of EMStreated animals (see Materials and Methods) were presented with $2 \mu \mathrm{L}$ of $100 \%$ benzaldehyde, and those animals that were closest to the spot of benzaldehyde after $60 \mathrm{~min}$ were

\section{口naive habituated $\mathbf{Q}$ dishabituated}

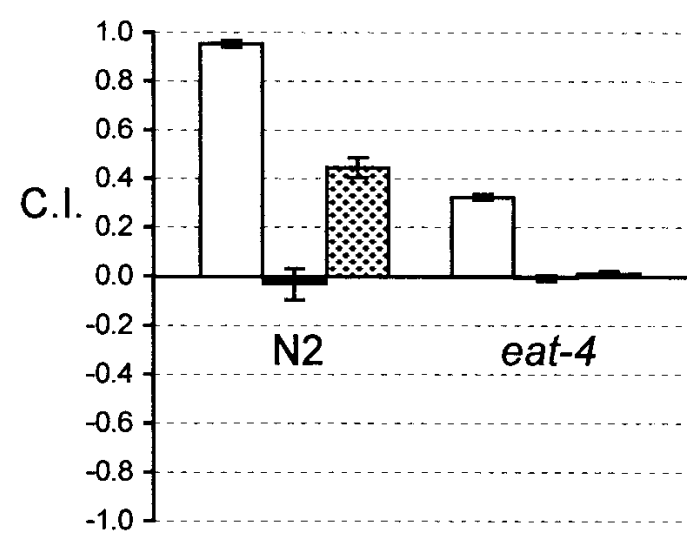

Figure 6 Olfactory behavior of eat-4(ky5) mutants. Naive, habituated (transferred in $2 \mathrm{~mL}$ of water and settled at $1 \mathrm{~g}$ ), and dishabituated (centrifuged twice at $65 \mathrm{~g}$ in $10 \mathrm{~mL}$ of water) wild-type $\mathrm{N} 2$, and eat-4(ky5) mutant animals were tested for their $1-\mathrm{h}$ approach to $1 \%$ benzaldehyde ( $n=9$ plates per point). isolated as potential persister mutants and used to establish lines. From the 64 primary lines isolated from the screen, one mutant line retained the persister phenotype in subsequent tests. Figure 7A shows the chemotaxis kinetics of this mutant line and wild-type animals in the presence of $100 \%$ benzaldehyde, including the significantly greater attraction displayed at the 60 -min time point that was the selection point in the screen. As expected, these animals also showed a deficit in habituation to benzaldehyde as measured in the two-plate assay. When this line was back-crossed twice to the parental N2 strain to generate a mutant line, designated UT20, the segregation of the habituation phenotype was consistent with a single, recessive mutation (see Materials and Methods). The naive approaches to $1 \%$ benzaldehyde by wild-type and UT20 animals were indistinguishable $\left(t_{6}=0.9, P<0.05\right.$; Fig. 7B). Although a significant response decrement resulted from benzaldehyde pre-exposure for both wild-type and mutant strains, UT20 animals exhibited a significantly greater attraction to benzaldehyde after preexposure compared with the approach by wild-type N2 animals $\left(t_{28}=4.1, P<0.05\right.$; Fig. $\left.7 \mathrm{~B}\right)$ showing that the UT20 animals habituated less. Additionally, UT20 animals showed decreased adaptation relative to N2s when tested with a second AWC-sensed odorant, isoamyl alcohol $\left(t_{13}=2.7\right.$, $P<0.05)$, although naive isoamyl alcohol approaches were at wild-type levels $\left(t_{6}=0.9, P<0.05\right)$. The adaptation to diacetyl (Fig. 7B), which is sensed by the AWA sensory neurons, was unaffected in UT20 animals $\left(t_{13}=0.4\right.$, $P>0.05)$.

The initial mutant strain (Fig. 7A) showed a higher weighted C.I. (W.C.I.) than the wild-type animals at the 10-min time point. It is possible that the apparent habituation deficit in UT20 could be due to an increased level of attraction to odorants, but this behavior is not seen in naive animals because of a ceiling effect in the assays for approach to $1 \%$ benzaldehyde. That is, the naive animals achieve near maximal C.I. scores, so any increased attraction in the mutant strain would go undetected in these assays. To address this issue, we compared the chemotactic scores of wild-type and backcrossed UT20 mutant animals to lower concentrations of benzaldehyde and isoamyl alcohol, where any increase in naive attraction would not be masked by a ceiling effect. There is no significant difference in the attractions displayed by wild-type and mutant animals to either of these odorants when tested to lower concentrations of the odorants (Fig. 7A). Additionally, 15-min approach scores with wild-type and backcrossed UT20 animals showed no difference in the initial level of attraction to $100 \%$ benzaldehyde (C.I. $=0.54 \pm 0.05$ for wild type and C.I. $=0.49 \pm 0.05$ for UT20; $t_{8}=0.8$, $P<0.05)$. This result suggests that if the original isolate did in fact have a higher naive level of attraction to $100 \%$ benzaldehyde, this phenotype did not co-segregate with the habituation phenotype through the two back-crosses, making

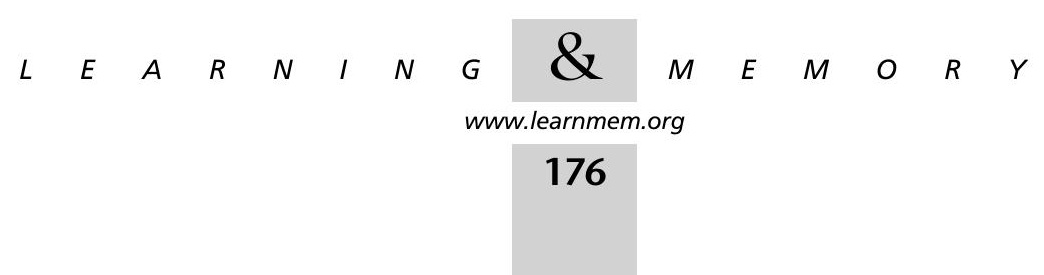



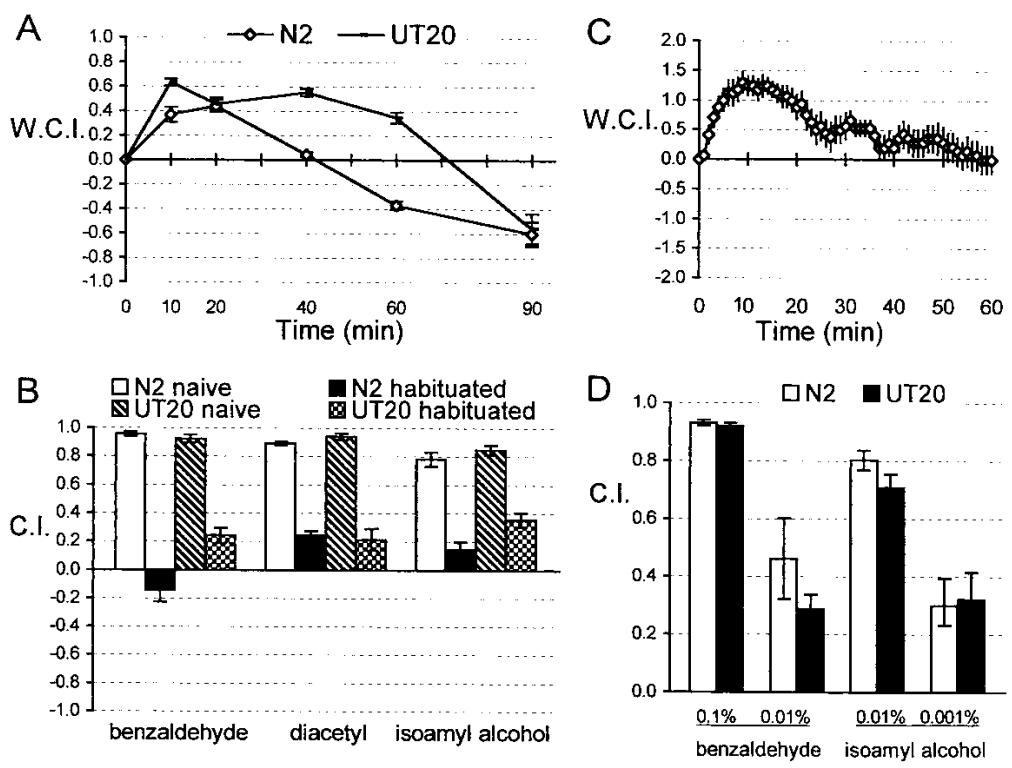

Figure 7 Characterization of UT20. (A) Population chemotaxis of the mutant strain (UT20) vs. the wild-type (N2) animals in response to $2 \mu \mathrm{L}$ of $100 \%$ benzaldehyde (N2 $n=12$ plates per point, UT20 $n=5$ plates per point). (B) Habituation to benzaldehyde, diacetyl, and isoamyl alcohol by N2 and UT20. Animals were exposed to $2 \mu \mathrm{L}$ of odorant for $1 \mathrm{~h}$, then tested for their 1-h approach to the same odorant diluted in ethanol to $1 \%$ for benzaldehyde and $0.1 \%$ for diacetyl and isoamyl alcohol (from left, $n=3,15,5,15,5,9,3,6,5,9,3$, and 6 plates). (C) Performance of single UT20 animals during $60 \mathrm{~min}$ of benzaldehyde chemotaxis $(n=17$ animals). (D) Naive approach to odorants. Naive wild-type and mutant animals were tested for their $1-\mathrm{h}$ approach to $0.1 \%$ and $0.01 \%$ benzaldehyde and $0.01 \%$ and $0.001 \%$ isoamyl alcohol ( $n=6$ plates per point).

it unlikely that a difference in the attraction to either benzaldehyde or isoamyl alcohol after habituation reflects a general increase in odorant sensitivity or the naive level of attraction.

Chemotaxis of single UT20 animals was analyzed by time-lapse video photography (Fig. 7C) confirming that the persister phenotype had co-segregated with the habituation phenotype through the back-crossing. A two-factor ANOVA over the 60-min assay showed a significant interaction of time and strain $\left(F_{1,59}=10.7, \mathrm{P}<0.05\right)$. Although the behavior of wild-type (Fig. 1C) and mutant (Fig. 7C) animals was the same for the first $30 \mathrm{~min}$ of the test (time $\mathrm{X}$ strain $F_{1,29}=0.8, P>0.05$ ), the C.I. for the UT20 mutants was significantly greater than that of the wild types from the 30-min time point until the end of the assay at $60 \mathrm{~min}$ (time $\mathrm{X}$ strain $F_{1,29}=3.9, P<0.05$ ), by which time the wild-type animals displayed a strong aversion (Fig. 1C). This result indicates a delay in the onset of the aversive response rather than an absence of the aversion, as population analysis of the back-crossed UT20 animals again confirmed the presence of an aversion after 90 min of exposure (C.I. $=-0.77 \pm 0.08, n=6$ plates) that did not differ from wild type $\left(\right.$ C.I. $=-0.97 \pm 0.07 ; t_{10}=2.2$, $P>0.05)$.

\section{DISCUSSION}

C. elegans displays a complex response when presented with a source of benzaldehyde in that the animals first approach then retreat from a high concentration of the odorant. Analysis of the odr-3(n2150) mutant confirmed that there are two genetically separable responses to benzaldehyde. Although the $o d r$-3 gene is required for the approach to benzaldehyde, it is not necessary for the expression of the aversive response. Therefore, the response of these animals is similar to the aversive response mechanism postulated in our model (Fig. 2). The UT20 animals did not display an aversion over the first $60 \mathrm{~min}$ of chemotaxis assays, yielding a curve similar to the attractive response depicted in Figure 2. Although these two mutants appear to provide a genetic double dissociation of the attractive and aversive responses to benzaldehyde (the arithmetic sum of the odr-3(n2150) and UT20 data is indistinguishable from the data for N2 animals), the significant aversion displayed by the UT20 mutants after prolonged exposure indicates that the aversive response has not been eliminated in this strain. Indeed, we interpret the UT20 phenotype as a decreased rate of habituation of the attractive response to benzaldehyde, resulting in a delay in the appearance of the aversive response. Therefore, screening for a delayed onset of the chemotactic aversion to benzaldehyde represents a high through-put alternative to the traditional two plate assay for identifying mutants with defects in olfactory learning and memory (Colbert and Bargmann 1995).

As the ODR-3 protein is required in the AWC neurons for chemotaxis to low concentrations of benzaldehyde, it is likely that the AWC neurons are required for the attractive response to a wide range of benzaldehyde concentrations, but not for the subsequent aversion. The existence of an AWC-independent aversion pathway is consistent with the conclusion drawn from studies with the diacetyl receptor, which indicated that individual olfactory neurons are either aversion-mediating or attraction-mediating (Troemel et al. 1997). The intact aversive response in odr-3(n2150) animals was somewhat surprising because $o d r-3$ is required for the function of the ASH neurons, which have been reported to mediate the aversion to benzaldehyde.

A second mutation that blocks ASH function, the osm9(ky10) allele, also failed to disrupt the wild-type chemotaxis, indicating that the aversion being assayed here may be ASH independent. It is significant that in the earlier studies of odorant avoidance, a capillary tube containing benzaldehyde was presented very close (a few millimeters) to the

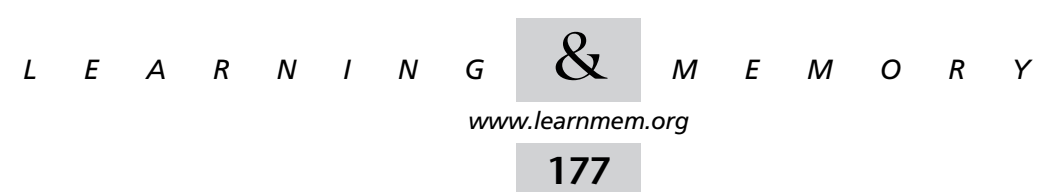


amphid opening at the tip of the animal, inducing a reflexive reversal in wild-type animals. This response was found to be impaired in osm-9(ky10) mutants and ASH-ablated animals (Troemel et al. 1995). In our assay, the animals are initially about $4 \mathrm{~cm}$ from the spot of odorant and they rarely approach closer than $1 \mathrm{~cm}$. This minimum separation may be indicative of the response elicited in the capillary tube assays and could explain how animals that were attracted to $100 \%$ benzaldehyde avoided the sodium azide that is used in standard chemotaxis assays to immobilize animals that reach the spot of odorant, hence yielding a negative C.I. at 60 min (Fig. 1A, 100\% benzaldehyde). Additionally, analysis of the odr-3(n2150) mutant suggested that the avoidance response in our assay requires odorant pre-exposure prior to affecting behavior, as naive animals showed no chemotactic bias at early time points in the assay. In contrast, the short-range aversive response measured in the previous assay appears robust in naive animals. Therefore, it appears that at least two different benzaldehyde-sensitive aversionmediating pathways exist in C. elegans, a short-range reflexive response requiring the ASH sensory neurons and a later, negative chemotaxis that is displayed by odr-3(n2150) and osm-9(ky10) animals and thus may be independent of the ASH neurons, although it remains possible that the lateonset chemotactic aversion to benzaldehyde is mediated by the ASH sensory neurons in an odr-3- and osm-9-independent manner.

Two other sensory neuron types known to be involved in aversive responses to volatile chemicals are the AWB and the ADL neurons, although neither of these has been implicated in responses to benzaldehyde. It is noteworthy that the odr-3 gene is expressed in the AWB neurons and $o d r$ 3(n2150) mutants were shown to be significantly defective in AWB-mediated avoidance of 2-nonanone. These mutants were also defective in the $o d r-10$ (diacetyl receptor)-dependent aversion to diacetyl, which was displayed when $o d r-10$ was expressed in the AWB neurons (Troemel et al. 1997). The ADL neurons are required for the short-range aversive response to at least one volatile chemical, octanol (Troemel et al. 1995). The osm-9 gene is expressed in the ADL neurons (Colbert et al. 1997), suggesting a role for the OSM-9 protein in the function of these neurons. However, mutations in either the odr-3 or the osm-9 genes did not affect the expression of the chemotactic aversion after habituation to benzaldehyde. Additionally, laser killing of individual amphid neuron pairs, including AWB or ADL, does not eliminate this chemotactic aversion (B. Kimmel and C. Bargmann, pers. comm.). These results suggest that the chemotactic aversion to benzaldehyde may involve a behaviorally uncharacterized chemosensory pathway or multiple, redundant sensory mechanisms.

The failure of the adp-1(ky20) allele to disrupt the aversive phase of benzaldehyde chemotaxis led us to reexamine the behavior of this mutant. Our analysis indicates that adp-1(ky20) increases the sensitivity of the animals to dishabituating stimuli. We have also shown that the previously reported adaptation to benzaldehyde in wild-type N2 animals can be dishabituated by a mild centrifugation step. This observation indicates that the decrement in the approach response is due to true habituation, rather than sensory fatigue (Jorgensen and Rankin 1997). Furthermore, the lack of cross habituation to diacetyl or butanone demonstrates the stimulus specificity of olfactory habituation in nematodes. We also observed an increased amount of habituation with increasing odorant concentration. The properties of benzaldehyde chemotaxis are in keeping with the most significant feature of habituation, that being the apparent redirection of attentional mechanisms, rather than a decreased attractive response caused by fatigue. To our knowledge, the adp-1(ky20) allele identifies the first mutation that specifically increases dishabituation.

The eat-4(ky5) mutation had an opposite effect compared with adp-1(ky20). Eat-4(ky5) rendered the animals insensitive to our dishabituation protocol, although the pleiotropic nature of this mutant, including greatly reduced naive chemotaxis scores, suggests that the lack of dishabituation in this strain could be the by-product of a more general physiological deficit in these animals. The eat- 4 gene encodes a homolog of a mammalian vesicular glutamate transporter (Bellocchio et al. 2000; Takamori et al. 2000) and has been shown to be necessary for glutamatergic neurotransmission in C. elegans (Lee et al. 1999). The deficiency of eat-4(ky5) mutants in the habituation and dishabituation of the tap-response has been interpreted as resulting from an inability to rapidly replenish glutamate levels at pre-synaptic sites and thereby support sustained neurotransmitter release (Rankin and Wicks 2000). The eat-4 gene does not appear to be expressed in the AWC neurons (Lee et al. 1999), and the effects of the eat-4(ky5) mutation on benzaldehyde chemotaxis may result from the inability of eat-4(ky5) mutants to support sustained glutamatergic neurotransmission in interneuron circuits downstream of AWC that are required for chemotaxis. If this is the case, then $C$. elegans sensory neurons must be able to isolate multiple sensory pathways to maintain the ability to adapt to one odorant while maintaining naive responses to others detected by the same sensory neuron (Colbert and Bargmann 1995, 1997). Alternatively, the depletion of glutamate levels by the eat-4(ky5) mutation may impose a more general effect on the nervous system that is not specific to the habituation and dishabituation we observe in wild-type animals.

Both the centrifugation force and the high-volume wash were required for maximal dishabituation. GPCRs are believed to mediate olfactory responses, and at least four different G-protein $\alpha$-subunit genes are expressed in the AWC neurons (Jansen et al. 1999). Recently, RGS (regulators of $\underline{G}$-protein signaling) proteins have been shown to

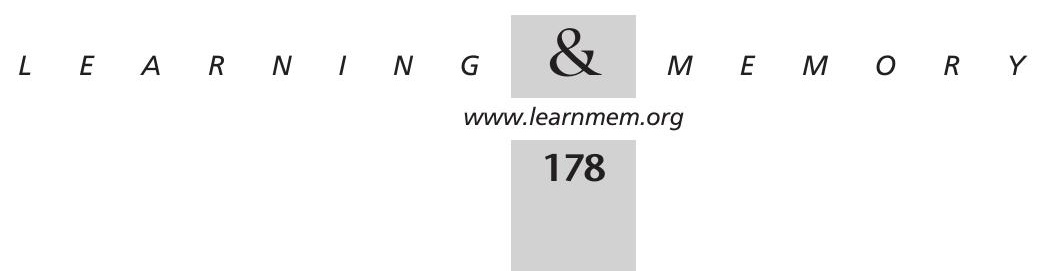


mediate abrupt changes in nematode behavior (Dong et al. 2000), providing a possible mechanism for the rapid recovery seen after dishabituation treatment. Alternatively, habituation may involve the reversible phosphorylation of signal transduction components involved in chemotaxis and perhaps the down-regulation of surface receptors by endocytosis, as is the case for adaptation involving nicotinic receptors (Waggoner et al. 2000). If receptor endocytosis is involved in benzaldehyde habituation, then dishabituation could result from the recycling of receptors to the cell surface by the fusion of receptor-containing vesicles with the plasma membrane. Interestingly, the trafficking of glutamate receptors, which are important for some forms of neuronal plasticity, has been shown to involve the activitydependent cycling of receptor molecules into and out of dendritic surface membranes (Ehlers 2000).

The isolation of mutant lines such as UT20 with altered timing of the transition from the attraction to the aversion to benzaldehyde should lead to the identification of additional genes involved in olfactory habituation. It is important to note, however, that further mapping experiments and rescue of the mutant phenotype by introduction of a wild-type transgene will be needed to confirm that a single genetic lesion is responsible for the behavioral phenotypes described here. The UT20 phenotype, affecting the response to AWC- (benzaldehyde, isoamyl alcohol) but not AWA- (diacetyl) sensed odorants, is distinct from those of previously described adaptation mutants in C. elegans, which are affected in their response to either benzaldehyde or isoamyl alcohol, but not both (Colbert and Bargmann 1995). The specificity of the dishabituation protocol, affecting benzaldehyde- but not isoamyl alcohol-pre-exposed animals, suggests that different underlying mechanisms may be involved in the responses to benzaldehyde (habituation) and isoamyl alcohol (adaptation), although both responses are compromised in UT20. It may be that isoamyl alcoholadapted animals are susceptible to dishabituation, but we have not found the appropriate conditions. Alternatively, UT20 may carry a mutation in a gene that is required for both habituation and adaptation. The observation that the same stimulus can induce either habituation or adaptation, depending on the protocol employed (Bernhard and van der Kooy 2000), suggests that these two processes do share some common components.

Analysis of the attraction-deficient $o d r-3(n 2150) \mathrm{mu}-$ tant showed a lag in the onset of the aversion to benzaldehyde, suggesting that this response may require prolonged odorant exposure for a process akin to sensitization to occur. Accounts of non-associative plasticity have been put forward in which response habituation results from the sensitization of an opposing response (Groves and Thompson 1970) and it is possible that the attractive response persists throughout the time of the assay but is overwhelmed by the strengthening of the aversive response. However, the ob- servation that the N2 animals achieve aversion scores comparable to the odr mutants that are defective in the attractive response argues that the wild-type animals do not retain a significant attraction after $90 \mathrm{~min}$ of exposure to benzaldehyde. Although the mechanisms underlying olfactory habituation in C. elegans are still unknown, work in mammalian systems has shown that olfactory adaptation can result from the direct down-regulation of components in the signal transduction pathways involved in the initial odorant detection, rather than the strengthening of an antagonistic response (Kurahashi and Menini 1997; Wei et al. 1998).

The data support our model (Fig. 2) in which two antagonistic chemotactic response pathways contribute to whole animal behavior. Such antagonistic systems have been shown for other nematode responses as well. Laser ablation studies have shown that thermotactic behavior results from the interplay of separable thermophilic and cryophilic circuits, which are mediated by the AIY and AIZ interneurons, respectively (Mori and Ohshima 1995). Similarly, tap-response behavior results from the competition between tap-sensitive reversal and acceleration controlling circuits, which habituate with different kinetics (Wicks and Rankin 1995). Our results suggest that benzaldehyde chemotaxis also relies on two separate sensory processes that exhibit significant plasticity and that the interplay between the two processes results in an apparent change in the motivational properties of benzaldehyde over time, such that an initially attractive stimulus becomes aversive with prolonged exposure. The tractability of C. elegans as an experimental organism promises that the analysis of chemotactic behavior will provide a valuable system for discovering the mechanisms underlying such behavioral plasticity.

\section{MATERIALS AND METHODS}

\section{Strains and General Methods}

Nematode strains were obtained from the Caenorbabditis Genetics Center at the University of Minnesota. All experiments used wellfed, gravid adults cultivated at $20^{\circ} \mathrm{C}$ under standard conditions (Wood et al. 1988). Escherichia coli strain OP50 was used in all experiments.

\section{Chemotaxis Assays}

All chemotaxis assays were performed with standard 100-mm petri dishes containing $6 \mathrm{~mL}$ of chemotaxis medium (CTX; $10 \mathrm{mM}$ MOPS [pH 7.2], $0.25 \% \mathrm{vol} / \mathrm{vol}$ Tween-20, $15 \mathrm{gm} / \mathrm{L}$ agar). Where necessary, odorants were diluted in ethanol and reported as percent by volume. Plates were sealed with a strip of parafilm around the edge during all odorant exposures. Standard, 1-h chemotaxis assays were performed according to the methods of Bargmann et al. (1993). Briefly, 15 min prior to the assay, $1 \mu \mathrm{L}$ of $1 \% \mathrm{NaN}_{3}$ was applied to the centers of two test spots that were $6 \mathrm{~cm}$ apart. This chemical acts as an anesthetic to immobilize any animals that reach the spot during the assay. One-hundred to two-hundred individuals were then placed at the center of the plate between the two spots and 1 $\mu \mathrm{L}$ of the test odorant was placed at one spot, and $1 \mu \mathrm{L}$ of ethanol

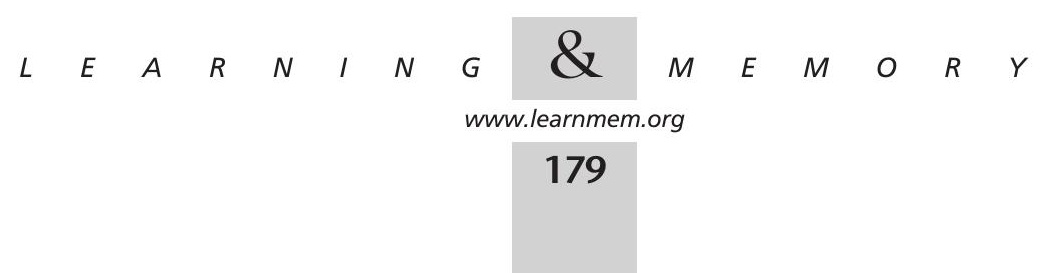


was applied to the other spot. After $1 \mathrm{~h}$ of chemotaxis, animals within $2 \mathrm{~cm}$ of either spot were counted, and a C.I. was calculated as the number of animals at the test spot minus the number of animals at the control spot, divided by the total number of animals on the plate. A positive C.I. indicated an attraction to the odor and a negative C.I. indicated an aversion.

For kinetic analysis of chemotaxis and for approaches to $100 \%$ sources of odorants a modified grid was used, and the $\mathrm{NaN}_{3}$ was omitted so that animals could leave a spot after an initial approach. This grid consisted of four parallel lines drawn $18 \mathrm{~mm}$ apart to divide the plate area into five sectors, symmetrical about the third. Two microliters of the odorant was placed on one of two small pieces of parafilm that had been placed at opposite edges of the agar. At the indicated time points, animals were immobilized by cooling the plates for $2 \mathrm{~min}$ at $-20^{\circ} \mathrm{C}$, and the plates were maintained at $4{ }^{\circ} \mathrm{C}$ until counting. The number of animals in sectors A-E, with the test odorant being in A, were counted, and a W.C.I. was calculated as $([2 \times$ no. in $A+$ no. in $B]-[2 \times$ no. in $\mathrm{E}+$ no. in $\mathrm{D}]$ )/(total number of animals on the plate), yielding a W.C.I. range of +2.0 to -2.0 . Those animals that crawled up the side of the plate were considered lost, and no attempt was made to count these. The score for each plate of 100-300 animals is one data point.

For standard adaptation assays, 500-1000 animals were placed on a CTX plate. Then, $2 \mu \mathrm{L}$ of the odorant was placed on a piece of parafilm on the lid of the plate, and the plate was sealed with parafilm and left inverted for $1 \mathrm{~h}$. Then, the animals were rinsed from the test plate in $\sim 2 \mathrm{~mL}$ of water and transferred to a conical centrifuge tube where they were allowed to settle to the bottom of the tube. After a few minutes, always $<5 \mathrm{~min}$, the concentrated animals were collected and transferred to a fresh CTX plate for testing. Modified, single-plate adaptation assays were performed as follows. Approximately 200 animals were placed on a CTX plate, and four $0.5-\mu \mathrm{L}$ spots of $100 \%$ benzaldehyde were placed at the four corners $(12,3,6$, and 9 o'clock positions) of the plate. This arrangement causes the animals to move toward the center of the plate by the end of $1 \mathrm{~h}$, presumably because that is the spot furthest from the benzaldehyde sources. After $1 \mathrm{~h}$ of adaptation, the parafilm pieces with the benzaldehyde spots were removed, the plate was left open to air out for 15-20 min and standard chemotaxis assays were then performed on the same plate.

For single-animal assays, individual animals were placed at the center of a petri dish and $2 \mu \mathrm{L}$ of $100 \%$ benzaldehyde was placed on one of two, 4-mm parafilm squares placed at opposite sides of the plate. Then, the plate was placed on top of a grid and chemotaxis was recorded for 60 min with a Sony TRV- 65 camcorder connected to a Panasonic AG4790 time-lapse video recorder. Animals were subsequently scored for their position every minute. Single-animal assays were terminated at $60 \mathrm{~min}$ because preliminary studies with $\mathrm{N} 2$ animals showed a basement effect by $60 \mathrm{~min}$. That is, they showed a strong aversion after the first $60 \mathrm{~min}$ of exposure, and the W.C.I. scores did not change in the 60- to 90-min time period. In both individual and population assays, the scores did not reach the theoretical W.C.I. minimum of -2.0 because individual animals occasionally wandered back into the areas closer to the benzaldehyde, and this fact is reflected in the cumulative W.C.I..

All C.I. and W.C.I. values are the mean and standard error of the mean calculated for multiple samples. $t$-Tests (Microsoft Excel 7.0) were used for two-way comparisons of statistical significance. ANOVAs were calculated by use of GB-STAT (Dynamic Microsystems).

\section{Mutagenesis and Screening}

Germ-line mutations were generated by treatment of $\mathrm{L} 4$ larvae with $50 \mathrm{mM}$ EMS for $4 \mathrm{~h}$ (Wood et al. 1988). Approximately 2,000 $\mathrm{F}_{1}$ progeny of 100 mutagenized animals were used for the isolation of $\sim 60,000 \mathrm{~F}_{2}$ animals. Then, these were tested in groups of about 300 per $10-\mathrm{cm}$ petri dish for their response to a single $2-\mu \mathrm{L}$ drop of $100 \%$ benzaldehyde. Because wild-type animals typically display an aversion by $60 \mathrm{~min}$ in these assays, we isolated animals that were still found within $2 \mathrm{~cm}$ of the benzaldehyde source after $1 \mathrm{~h}$. Of the 64 individual animals isolated from the primary screen, only one established a line, UT20, that consistently showed this phenotype in subsequent tests. The mutant line was back-crossed twice to the wild-type, N2, strain used for mutagenesis. From the first backcross, 9 of 45 (20\%) $\mathrm{F}_{2}$ clonal lines displayed the habituation phenotype, as did 5 of the $12(40 \%) \mathrm{F}_{2}$ lines analyzed from the second back-cross.

\section{ACKNOWLEDGMENTS}

We thank Dr. Cori Bargmann for sharing unpublished results. We also thank Sue Runciman, Nilo Kaviani, and Rachel Long for their excellent technical assistance. This work was supported by the Natural Sciences and Engineering Research Council of Canada.

The publication costs of this article were defrayed in part by payment of page charges. This article must therefore be hereby marked "advertisement" in accordance with 18 USC section 1734 solely to indicate this fact.

\section{REFERENCES}

Bargmann, C.I. 1998. Neurobiology of the Caenorhabditis elegans genome. Science 282: 2028-2033.

Bargmann, C.I. and Mori, I. 1997. Chemotaxis and thermotaxis. In C. elegans II. (ed., D.L. Riddle, T. Blumenthal, B.J. Meyer, and J.R. Priess), pp. 645-677. Cold Spring Harbor Laboratory Press, Cold Spring Harbor, NY.

Bargmann, C.I. and Kaplan, J.M. 1998. Signal transduction in the Caenorhabditis elegans nervous system. Annu. Rev. Neurosci. 21: 279-308.

Bargmann, C.I., Thomas, J.H., and Horvitz, H.R. 1990. Chemosensory cell function in the behavior and development of Caenorhabditis elegans Cold Spring Harbor Symp. Quant. Biol. 55: 529-538.

Bargmann, C.I., Hartieg, E., and Horvitz, H.R. 1993. Odorant selective genes and neurons mediate olfaction in C. elegans. Cell 74: 515-527.

Bellocchio, E.E., Reimer, R.J., Fremeau, R.T., and Edwards, R.H. 2000. Uptake of glutamate into synaptic vesicles by an inorganic phosphate transporter. Science 289: 957-960.

Bernhard, N. and van der Kooy, D. 2000. A behavioral and genetic dissection of two forms of olfactory plasticity in Caenorbabditis elegans: Adaptation and habituation. Learn. Mem. 7: 199-212.

Colbert, H.A. and Bargmann, C.I. 1995. Odorant-specific adaptation pathways generate olfactory plasticity in C. elegans. Neuron 14: 803-812.

-1997. Environmental signals modulate olfactory acuity, discrimination and memory in Caenorhabditis elegans. Learn. Mem. 4: 179-191.

Colbert, H.A., Smith, T.L. and Bargmann, C.I. 1997. OSM-9, a novel protein with structural similarity to channels, is required for olfaction, mechanosensation, and olfactory adaptation in Caenorbabditis elegans. J. Neurosci. 17: 8259-8269.

Dong, M-Q., Chase, D., Patikoglou, G.A. and Koelle, M.R. 2000. Multiple RGS proteins alter neural $G$ protein signaling to allow C. elegans to rapidly change behavior when fed. Genes \& Dev. 14: 2003-2014.

Driscoll, M. and Kaplan, J. 1997. Mechanotransduction. In C. elegans II. (ed., D.L. Riddle, T. Blumenthal, B.J. Meyer, and J.R. Priess), pp. 
645-677. Cold Spring Harbor Laboratory Press, Cold Spring Harbor, NY.

Ehlers, M.D. 2000. Reinsertion or degradation of AMPA receptors determined by activity-dependent endocytic sorting. Neuron 28: $511-525$.

Groves, P.M. and Thompson, R.F. 1970. Habituation: A dual-process theory. Psychol. Rev. 77: 419-450.

Jansen, G., Thijssen, K.L., Werner, P., van der Horst, M., Hazendonk, E., and Plasterk, R.H. 1999. The complete family of genes encoding G proteins of Caenorbabditis elegans. Nat. Genet. 4: 414-419.

Jorgensen, E.M. and Rankin, C.H. 1997. Neural plasticity. In C. elegans II. (ed., D.L. Riddle, T. Blumenthal, B.J. Meyer, and J.R. Priess), pp. 769-790. Cold Spring Harbor Laboratory Press, Cold Spring Harbor, NY.

Kaplan, J.M. and Horvitz, H.R. 1993. A dual mechanosensory and chemosensory neuron in Caenorhabditis elegans. Proc. Natl. Acad. Sci. 86: 4372-4376.

Kurahashi, T. and Menini, A. 1997. Mechanism of odorant adaptation in the olfactory receptor cell. Nature 385: 725-729.

Lee, Y.N.L., Sawin, E.R., Chalfie, M., Horvitz, H.R., and Avery, L. 1999. EAT-4, a homolog of a mammalian sodium-dependent inorganic phosphate cotransporter, is necessary for glutamatergic neurotransmission in Caenorhabditis elegans. J. Neurosci 19: $159-167$.

Mori, I. and Ohshima, Y. 1995. Neural regulation of thermotaxis in Caenorhabditis elegans. Nature 376: 344-348.

Rankin, C.H. and Wicks, S.R. 2000. Mutations in the Caenorhabditis elegans brain-specific inorganic phosphate transporter eat- 4 affect habituation of the tap-withdrawal response without affecting the response itself. J. Neurosci. 20: 4337-4344.

Roayaie, K., Crump, J.G., Sagasti, A., and Bargmann, C.I. 1998. The G-alpha protein ODR-3 mediates olfactory and nociceptive function and controls cilium morphogenesis in C. elegans olfactory neurons. Neuron 20: 55-67.

Robertson, H.M. 1998. Two large families of chemoreceptor genes in the nematodes Caenorhabditis elegans and C. briggsae reveal extensive gene duplication, diversification, movement and intron loss. Genome Res. 8: 449-463.

Sengupta, P., Chou, J.H., and Bargmann, C.I. 1996. odr-10 encodes a seven transmembrane domain olfactory receptor required for responses to the odorant diacetyl. Cell 84: 899-909.

Takamori, S., Rhee, J.S., Rosenmund, C., and Jahn, R. 2000. Identification of a vesicular glutamate transporter that defines a glutamatergic phenotype in neurons. Nature 407: 189-194.

Tominaga, M., Caterina, M.J., Malmberg, A.B., Rosen, T.A., Gilbert, H., Skinner, K., Raumann, B.E., Basbaum, A.I., and Julius, D. 1998. The cloned capsaicin receptor integrates multiple pain-producing stimuli. Neuron 21: 531-543.

Troemel, E.R., Kimmel, B.E., and Bargmann, C.I. 1997. Reprogramming chemotaxis responses: Sensory neurons define olfactory preferences in C. elegans. Cell 91: 161-169.

Troemel, E.R., Chou, J.H., Dwyer, N.D., Colbert, H.A., and Bargmann, C.I 1995. Divergent seven transmembrane receptors are candidate chemosensory receptors in C. elegans. Cell 83: 207-218.

Waggoner, L.E., Dickinson, K.A., Poole, D.S., Tabuse, Y., Miwa, J., and Schafer, W.R. 2000. Long-term nicotine adaptation in Caenorhabditis elegans involves PKC-dependent changes in nicotinic receptor abundance. J. Neurosci. 20: 8802-8811.

Wei, J., Zhao, A.Z., Chan, G.C.K., Baker, L.P., Impey, S., Beavo, J.A., and Storm, D.R. 1998. Phosphorylation and inhibition of olfactory adenylyl cyclase by CaM kinase II in neurons: a mechanism for attenuation of olfactory signals. Neuron 21: 495-504.

Wicks, S.R. and Rankin, C.H. 1995. Integration of mechanosensory stimuli in Caenorhabditis elegans. J. Neurosci. 15: 2434-2444.

Wood, W.B. and Community of C. elegans Researchers. 1988. The nematode Caenorhabditis elegans. Cold Spring Harbor Laboratory Press, Cold Spring Harbor, NY.

Received August 31, 2000; accepted in revised form March 13, 2001.

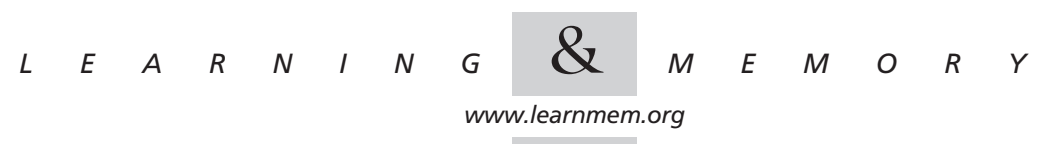




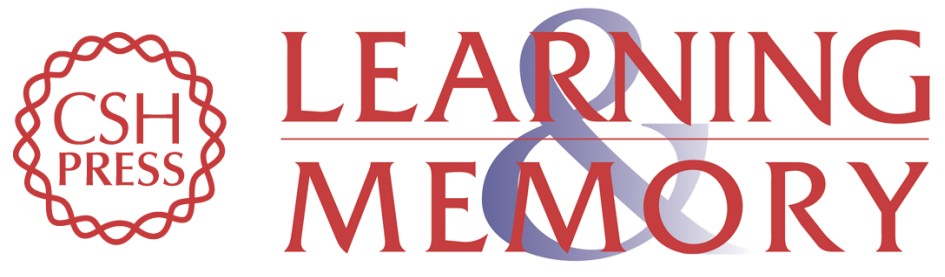

\section{Regulation of Distinct Attractive and Aversive Mechanisms Mediating Benzaldehyde Chemotaxis in Caenorhabditis elegans}

William M. Nuttley, Singh Harbinder and Derek van der Kooy

Learn. Mem. 2001, 8:

Access the most recent version at doi:10.1101//m.36501

References This article cites 27 articles, 12 of which can be accessed free at: http://learnmem.cshlp.org/content/8/3/170.full.html\#ref-list-1

License

Email Alerting Receive free email alerts when new articles cite this article - sign up in the box at the Service top right corner of the article or click here. 